\title{
High Light-Extraction GaN-Based Vertical LEDs With Double Diffuse Surfaces
}

\author{
Ya-Ju Lee, Hao-Chung Kuo, Senior Member, IEEE, Tien-Chang Lu, and Shing-Chung Wang, Senior Member, IEEE
}

\begin{abstract}
High light-extraction (external quantum efficiency $\sim 40 \%$ ) 465-nm GaN-based vertical light-emitting diodes (LEDs) employing double diffuse surfaces were fabricated. This novel LED structure includes one top transmitted diffuse surface and another diffuse omnidirectional reflector (ODR) on the bottom of a LED chip. The diffusive ODR consists of a roughened p-type GaN layer, an indium-tin-oxide (ITO) low refractive index layer, and an Al layer. The surface of the p-type GaN-layer was naturally roughened while decreasing the growth temperature to $800{ }^{\circ} \mathrm{C}$. After flip-bonding onto a Si substrate by AuSn eutectic metal and laser lift-off processes to remove the sapphire substrate, an anisotropic etching by dilute potassium hydroxide (KOH) was employed on the $N$-face $n$-GaN layer to obtain transmitted diffuse surfaces with hexagonal-cone morphology. The double diffused surfaces LEDs show an enhancement of $56 \%$ and $236 \%$ in light output power compared to single side diffused surface and conventional LEDs, respectively. The devices also show a low leakage current in the order of magnitude of $10^{-8} \mathrm{~A}$ at $-5 \mathrm{~V}$ and a calculated external quantum efficiency of about $40 \%$. The high scattering efficiency of double diffused surfaces could be responsible for the enhancement in the device light output power.
\end{abstract}

Index Terms-Double diffuse surfaces, GaN, light-emitting diodes (LEDs), light-extraction efficiency.

\section{INTRODUCTION}

W IDE BANDGAP light-emitting diodes (LEDs), that are III-Nitride, ranging from ultraviolet to the short-wavelength part of the visible spectrum have been intensely developed in the past ten years [1], [2]. Recently, as the brightness of GaN-based LEDs has increased, applications such as traffic signals, backlight for cell phone, and short-haul communications have become possible [3]. However, as for the replacement of conventional fluorescent lighting source with solid-state lighting, it still needs a great effort for improving the lightextraction efficiency as well as internal quantum efficiency of LEDs. Considering the large difference of the refractive index between $\mathrm{GaN}$ ( $\mathrm{n} \sim 2.5$ ) and air, the critical angle for light extraction is only about $23^{\circ}$, i.e., approximately $4 \%$ of light from

\footnotetext{
Manuscript received March 7, 2006; revised August 8, 2006. This work was supported in part by the National Science Council, Taiwan, R.O.C., under Contracts NSC 94-2120-M009-007 and NSC 94-2752-E009-007-PAE.

Y. J. Lee is with the Department of Photonic and Institute of Electro-Optical Engineering, National Chiao Tung University, Hsinchu 300, Taiwan, R.O.C., and also with the R\&D Division, Epistar Company, Ltd., Hsinchu 300, Taiwan, R.O.C. (e-mail: yjlee.eo92g@nctu.edu.tw).

H. C. Kuo, T. C. Lu, and S. C. Wang are with the Department of Photonic and Institute of Electro-Optical Engineering, National Chiao Tung University, Hsinchu 300, Taiwan, R.O.C. (e-mail: hckuo@faculty.nctu.edu.tw; timtclu@faculty.nctu.edu.tw; scwang@mail.nctu.edu.tw)

Color versions of Figs. 1, 2, and 5-7 are available online at http://ieeexplore. ieee.org.

Digital Object Identifier 10.1109/JQE.2006.883468
}

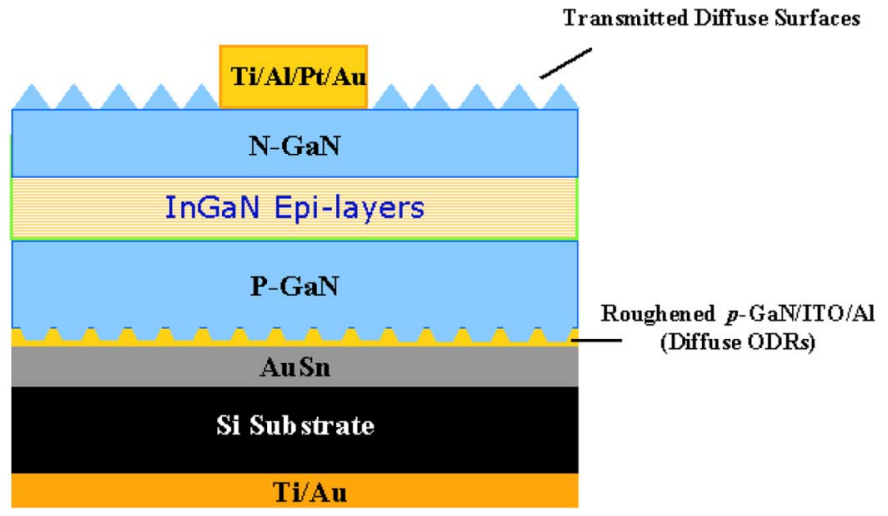

Fig. 1. Schematic cross section of a LED with double diffuse surfaces.

the active region can escape per surface. The light outside the escape cone is guided between sapphire substrate and the surrounding air, and then reabsorbed by active layers or electrodes. Therefore, there is much room for improving the light extraction efficiency. Many approaches have been proposed to obtain high light-extraction efficiency including shaping of LEDs [4], [5], patterned sapphire substrates [6], roughening of the top LED surface [7], and introducing highly reflective omnidirectional reflectors (ODRs) [8]. Recently, Kim et al. proposes a novel structure using diffuse ODRs, enhancing the reflected power by two orders of magnitude for a roughened reflector surface compared with a planar surface [9]. According to their investigation, guided light is reflected diffusively by the roughened reflector, thus adding the light-extraction efficiency. In this work, we present a LED structure adopting double diffused-surfaces - one top transmitted diffuse surface and another diffuse ODR surface on the bottom of a LED chip. According to this design, guided light scattered by bottom diffuse ODRs can be again scattered to angles inside the escape cone by top diffuse transmittance surfaces to achieve a high light external efficiency.

\section{DEVICE FABRICATION}

A schematic cross section image of a GaN-based LED with double diffuse surfaces is shown in Fig. 1. GaN-based LEDs used in this study were grown using a low-pressure metal-organic chemical vapor deposition (Aixtron 2600G) system onto the $C$-face (0001) 2"-diamerter sapphire substrate. The LED layer-structure comprised a 30-nm-thick GaN nucleation layer grown at $520{ }^{\circ} \mathrm{C}$, a $2-\mu$ m-thick undoped $\mathrm{GaN}$ layer grown at $1050{ }^{\circ} \mathrm{C}, 2$ - $\mu$ m-thick Si-doped n-type $\mathrm{GaN}$ cladding layer grown at $1050{ }^{\circ} \mathrm{C}$, an un-intentionally doped active region of 465-nm with five periods InGaN-GaN multiple quantum wells (MQWs) grown at $700{ }^{\circ} \mathrm{C}$, and a $0.4-\mu \mathrm{m}$-thick $\mathrm{Mg}$-doped p-type GaN cap-layer. Two different p-type GaN 
cap layers were used in this study. Samples A and B were grown at $1050{ }^{\circ} \mathrm{C}$ and $800{ }^{\circ} \mathrm{C}$, respectively. At such a low grown temperature of $\mathrm{GaN}$ layers (i.e., $800{ }^{\circ} \mathrm{C}$ ), large number of hexagonal pits can be seen on the top surface of the LED surface. In order to measure the change of surface roughness of p-type $\mathrm{GaN}$ surface caused by grown temperature, atomic force microscopy (AFM) measurements were performed in this report. After subsequent depositions of a 200-nm-thick indium-tin-oxide (ITO, $\mathrm{n} \sim 1.7$ at 465-nm) low-refractive-index layer and a 500-nm-thick Al mirror layer on top of LEDs, the p-side surface of sample A could be served as specular ODRs (flat $p$ - type GaN-ITO-Al) [15] and that of sample B could be served as diffuse ODRs (roughened $p-$ type GaN-ITO-Al) [9]. Thick Au was deposited on both samples followed by $\mathrm{Sn}$ evaporation in a thermal evaporator. The wafer was then flipped and bonded to an Au-coated $\mathrm{Si}$ submount at a temperature of $350{ }^{\circ} \mathrm{C}$, resulting in an alloy of $\mathrm{Au}$ and $\mathrm{Sn}$ to form adhesion between the LED wafers and $\mathrm{Si}$ submounts. A KrF excimer laser at a wavelength of $248 \mathrm{~nm}$ with a pulsewidth of $25 \mathrm{~ns}$ was then used to remove the sapphire substrate [14]. The laser with a beam size of $1.2 \mathrm{~mm} \times 1.2 \mathrm{~mm}$ was incident from the backside of the sapphire substrate onto the sapphire-GaN interface to decompose $\mathrm{GaN}$ into $\mathrm{Ga}$ and $\mathrm{N}_{2}$. In this process, the beam size of $\mathrm{KrF}$ laser was larger than that of the size of the LEDs. Therefore, the laser irradiation on the interface of sapphire and $\mathrm{GaN}$ was uniform. After laser lift-off process, the remaining $\mathrm{GaN}$ epitaxial film on the $\mathrm{Si}$ submount displayed a $N$-face (000-1) surface. The remaining Ga droplets on the transferred $\mathrm{GaN}$ surface were removed by etching in a $\mathrm{HCl}$ solution. Next, the transferred $\mathrm{GaN}$ was thinned until the $\mathrm{Si}$-doped $\mathrm{GaN}$ was exposed. An n-contact composed of Ti-Al-Pt-Au was formed on the exposed $N$-face $\mathrm{n}-\mathrm{GaN}$ and p-contact composed of $\mathrm{Ti}-\mathrm{Au}$ was deposited on the Si-submount. Finally, in order to roughen the top of surface, dilute potassium hydroxide $(\mathrm{KOH})$ was used as etching solution at $80{ }^{\circ} \mathrm{C}$. After wet etching, hexagonal cones can be observed on the $N$-face $n-G a N$ surface. The conventional LEDs with the same emitting wavelength as above two samples were also used as a reference to evaluate the optical and electrical properties of double diffused surfaces LEDs, where the p-type GaN cap layer of conventional LEDs was grown at $1050{ }^{\circ} \mathrm{C}$. The as-grown LED epitaxial layers were partially etched until the n-type GaN layers were exposed. $\mathrm{Ni}$-Au transparent contact layers were subsequently evaporated onto the $\mathrm{p}-\mathrm{GaN}$ cap layers to serve as the transparent contact layers (TCLs). A thick Au layer was then deposited onto part of the TCL to serve as the p-electrodes. Ti-Al-Pt-Au contacts were deposited onto the exposed n-GaN layers to serve as the n-electrodes. All LEDs were fabricated with the size of $350 \times 350 \mu \mathrm{m}^{2}$. The typical current-voltage $(I-V)$ measurements were performed using a high current measure unit (KEITHLEY 240). The light output power of the LEDs was measured on TO-can without epoxy resin and used an integrated sphere with a calibrated power meter.

\section{RESUlTS AND DISCUSSION}

Fig. 2 shows AFM images of p-type GaN surface grown at (a) $1050{ }^{\circ} \mathrm{C}$ and (b) $800{ }^{\circ} \mathrm{C}$. With the variation of grown temperature, the root-mean-square (rms) roughness of p-type (a)



(b)

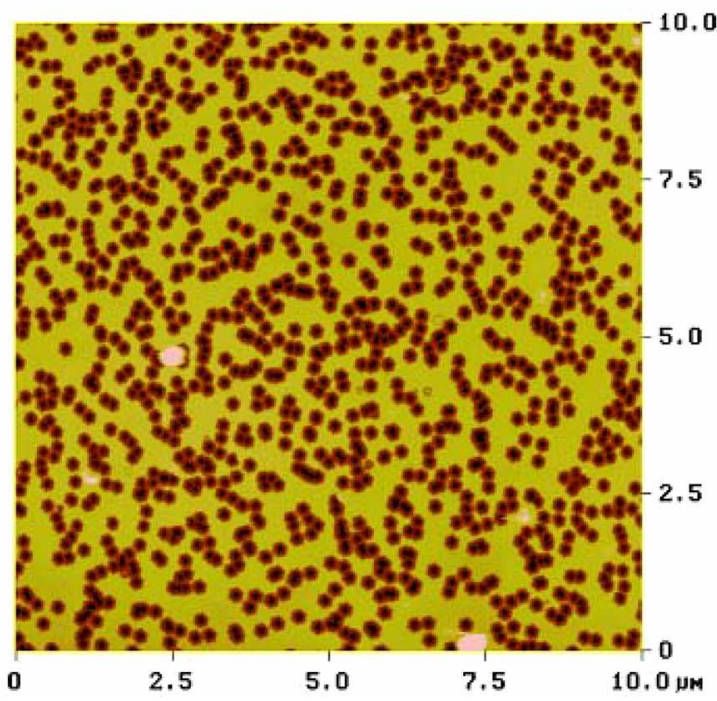

Fig. 2. AFM images of p-type $\mathrm{GaN}$ surface grown at (a) $1050{ }^{\circ} \mathrm{C}$ and (b) $800^{\circ} \mathrm{C}$. The rms roughness is (a) 2.3 and (b) $129.3 \mathrm{~nm}$.

$\mathrm{GaN}$ increases from (a) 2.3 to (b) $129.3 \mathrm{~nm}$, respectively. According to Fig. 2 (b), hexagonal V-shape pits distributed randomly and the surface density was estimated to be about $1.28 \times 10^{9} / \mathrm{cm}^{2}$. Fig. 3(a) and (b) shows the cross-sectional transmission electron microscope (TEM) images of sample A and sample B, respectively. In Fig. 3(a), the top surface of p-type $\mathrm{GaN}$ was quite flat, as can be seen in conventional LEDs; however, lots of hexagonal V-shape pits was observed on p-type GaN surface of sample B, as shown in Fig. 3(b). The diameter of hexagonal V-shape pits ranged from 200 to $400 \mathrm{~nm}$, and the depth of pits was about $300 \mathrm{~nm}$. Therefore, light emitted from LED active region could be scattered due to these randomly distributed hexagonal V-shape pits. Fig. 3(c) is an enlarged TEM image of one hexagonal $\mathrm{V}$-shape pit. As can be seen in this figure, the hexagonal $\mathrm{V}$-shape pit originated from threading dislocations and there is a thick dark band along the V-groove, being indicative of thickness variation. The formation of these 
(a)

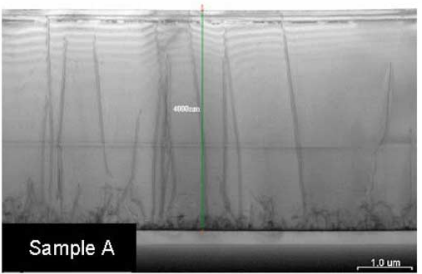

(b)

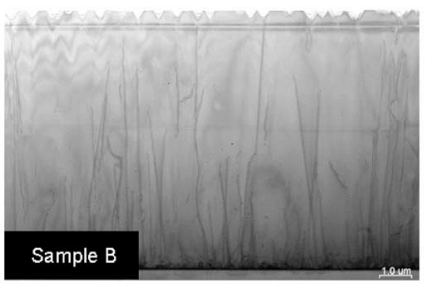

(c)

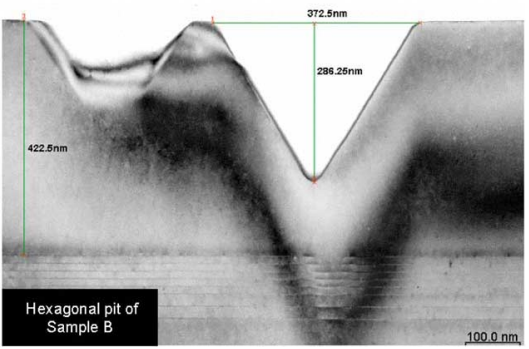

Fig. 3. Cross-sectional transmission electron microscope (TEM) images of (a) flat $\mathrm{p}$-GaN surface (sample A) and (b) hexagonal V-shape roughened p-GaN surface (sample B). (c) is an enlarged TEM image of one hexagonal V-shape pit.

hexagonal V-shape pits could be attributed to the fact that $\mathrm{Ga}$ atoms might not have enough energy to migrate to proper sites. Thus, lateral growth rate of $\mathrm{GaN}$ will become smaller [10]-[13]. Fig. 4(a) shows the top-view scanning electron micrograph (SEM) image of $N$-face n-GaN surface after $\mathrm{KOH}$ etching for $3 \mathrm{~min}$. Shown in Fig. 4(b) was SEM image taken at an angle of 70 degrees from the perpendicular direction of the etched surface. According to this figure, the base and height of hexagonal cones are both about $400-600 \mathrm{~nm}$. It should be noted that $n-G a N$ surfaces of both sample $A$ and $B$ exhibit the same surface morphology since they were etched by $\mathrm{KOH}$ solution at the same time. Therefore, $N$-face n-GaN surfaces with hexagonal cones features were quite suitable for serving as transmitted diffuse surfaces and an excellent transmitted capability of this roughened morphology had been demonstrated [7]. Fig. 5(a) shows the light output power ( $L-I$ curve) of sample A, sample B and conventional LEDs. The sample B, the LED with double diffuse surfaces, and sample A, the LED with flat ODRs, produced much higher light output as compared with that of conventional LEDs under all our measurement condition. For instance, the light output powers at $20 \mathrm{~mA}$ of sample A, sample B, and conventional LEDs are 13.6, 21.3, and $9 \mathrm{~mW}$, respectively. Therefore, the light output at $20 \mathrm{~mA}$ of the sample B increases by $236 \%$ as compared with that of conventional LED and increases by $56 \%$ as compared with that of sample A. The calculated external quantum efficiency of our proposal LEDs with double diffuse surfaces is about $40 \%$ at (a)

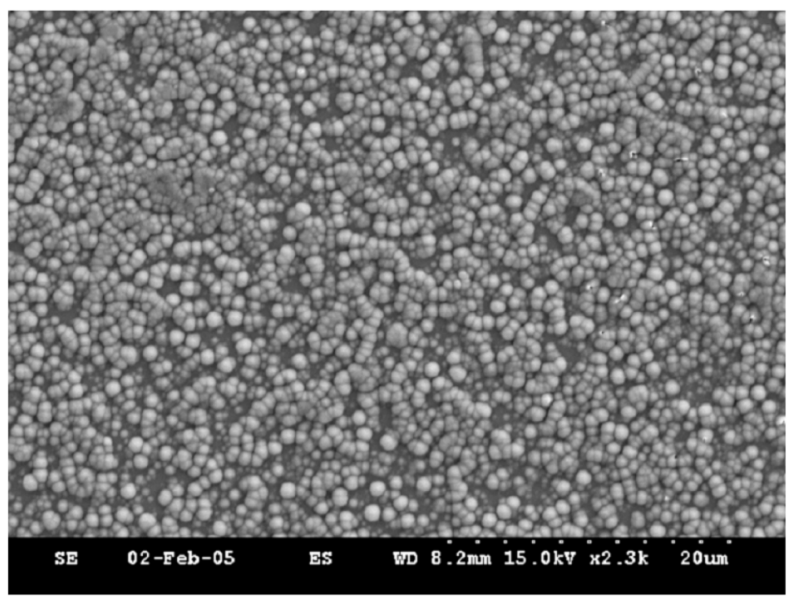

(b)

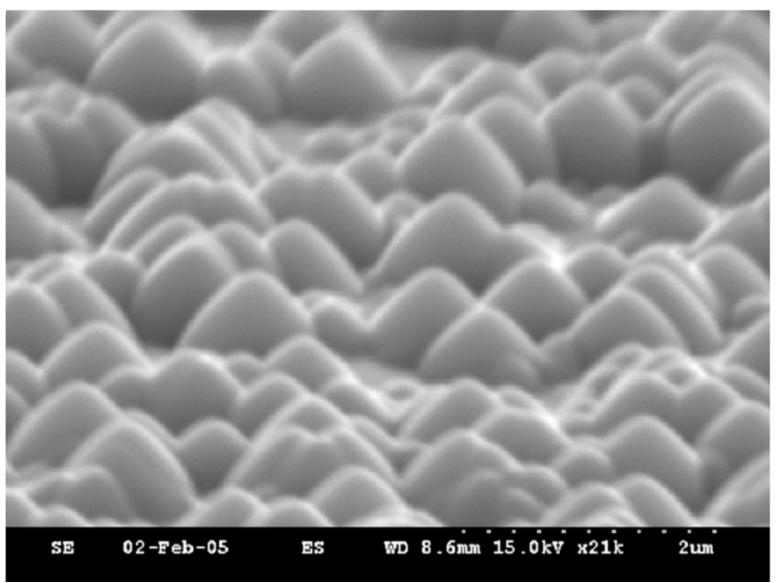

Fig. 4. SEM images of the $\mathrm{N}$-face $\mathrm{GaN}$ etch morphology after an etch time of 3 min by $\mathrm{KOH}, 80^{\circ} \mathrm{C}$. (a) Top-view and (b) is taken at an angle of 70 degrees from the perpendicular to the etched surface.

$20 \mathrm{~mA}(\lambda \sim 465 \mathrm{~nm})$, which could compete with structures of state of the art [16]. Fig. 5(b) shows light output patterns of sample A, sample B and conventional LED at $20 \mathrm{~mA}$. It is clear from the results that the EL intensities of sample $B$ were larger than those of sample A and conventional LEDs. According to this figure, view angles (half-center brightness or $50 \%$ of the full luminosity) of sample A and sample B are almost the same, i.e., $120^{\circ}$; however, the overall integrated area of EL intensities of sample B is still larger than that of sample A. Besides, although view angles of conventional LEDs is larger than that of sample A and sample B, i.e., $140^{\circ}$, due to the existing of transparent sapphire substrate; the enhancement of EL intensity by adopting double diffuse surfaces scheme is more remarkable, as compared that with conventional LEDs. The improvement in emitted light extraction efficiency is considered as a consequence of high light scattering efficiency by employing double diffuse surfaces. To understand the light-output enhancement of the LEDs made with double diffuse surfaces, the propagation of light emitted is schematically shown in Fig. 6. Fig. 6(a) shows a simple optical ray trace diagram of a conventional LED. In this case, the guided light emitting with an angle larger 
(a)

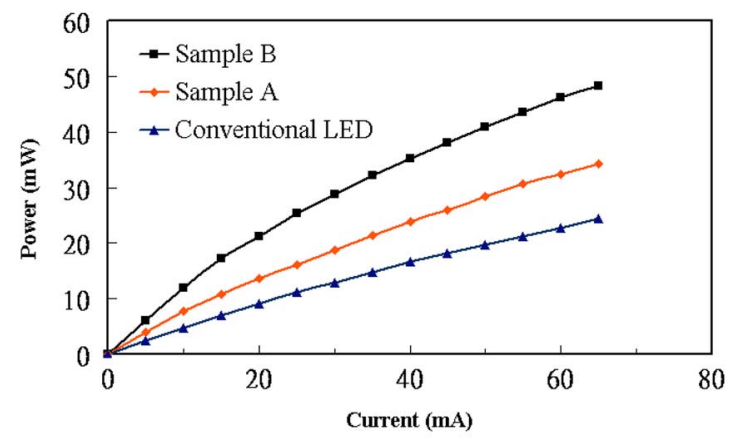

(b)

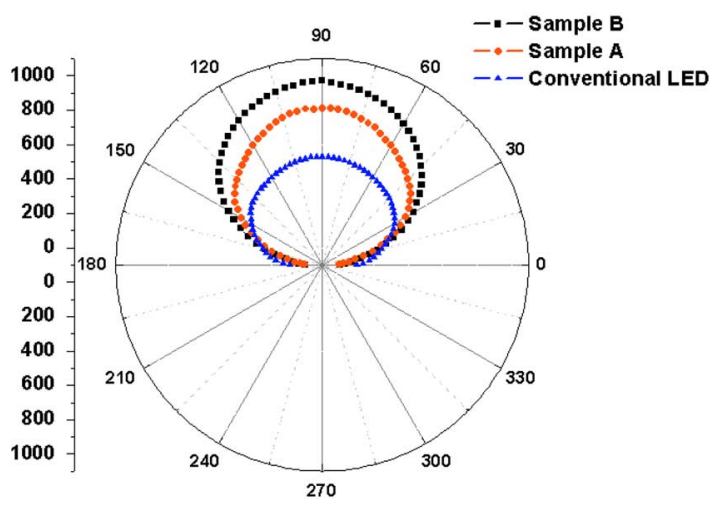

Fig. 5. (a) Output power of sample A, sample B, and conventional LEDs measured by an integral-sphere as a function of a forward dc current. (b) Light output patterns of sample A, sample B, and conventional LEDs.

than the critical angle would be trapped between sapphire substrate and the surrounding air, and finally be vanished through absorption of active layers or electrodes. Fig. 6(b) shows the case of sample A by employing transmitted diffuse surfaces on top and flat ODRs on bottom of LED chips. According to this figure, guided light could be extracted outside LED chips by scatterings at transmitted diffuse surfaces; therefore in this case, the escaping probability of photons is larger as compared with that of conventional LEDs. However, guided light needs multiple reflections by the bottom diffuse omnidirectional surfaces before being scattered again by the top transmitted diffuse surfaces. When we replace flat ODRs with diffuse or roughened surfaces, as shown in Fig. 6(c), the situation is quite different. In Fig. 6(c), guided light emitted towards to diffuse ODRs is scattered. The scattering light will be scattered again by top transmitted diffuse surfaces, adding total probability for photons to escape outside LED chips. Therefore, higher guided light scattering efficiency was achieved by adopting double diffuse surfaces scheme, as shown in Fig. 6(c). It's the reason why sample B demonstrated the best light output compared to sample A and conventional LEDs in all our measurement conditions.

As for the electrical characteristics, all LEDs demonstrate forward voltages of $3.2 \mathrm{~V}$ at $20 \mathrm{~mA}$, which is a typical and acceptable value while comparing to other devices with same chip size $\left(350 \times 350 \mu \mathrm{m}^{2}\right)$. Another important electrical property needed to certify is the reverse leakage current, since the

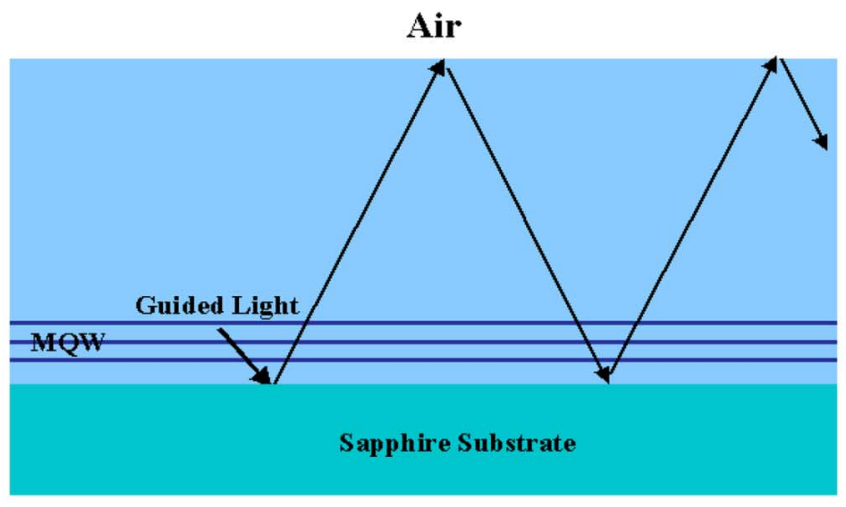

(a)

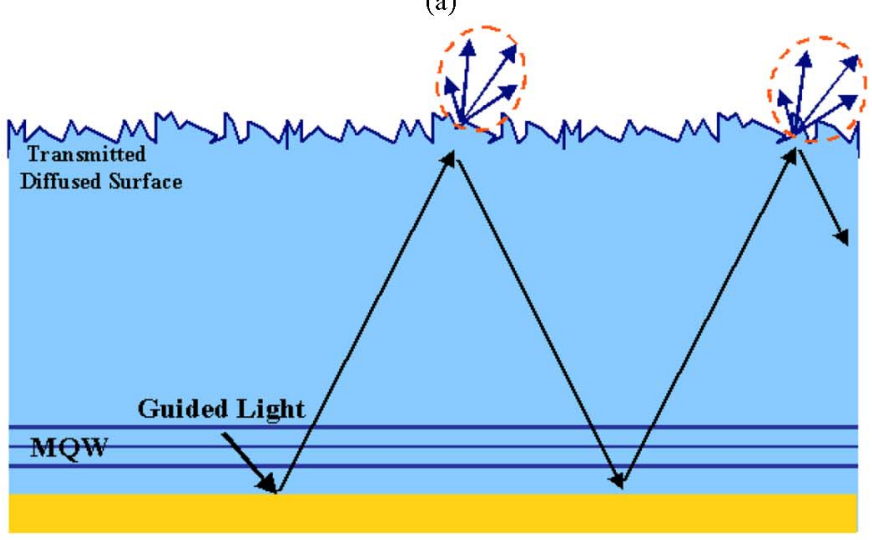

Flat Reflector

(b)

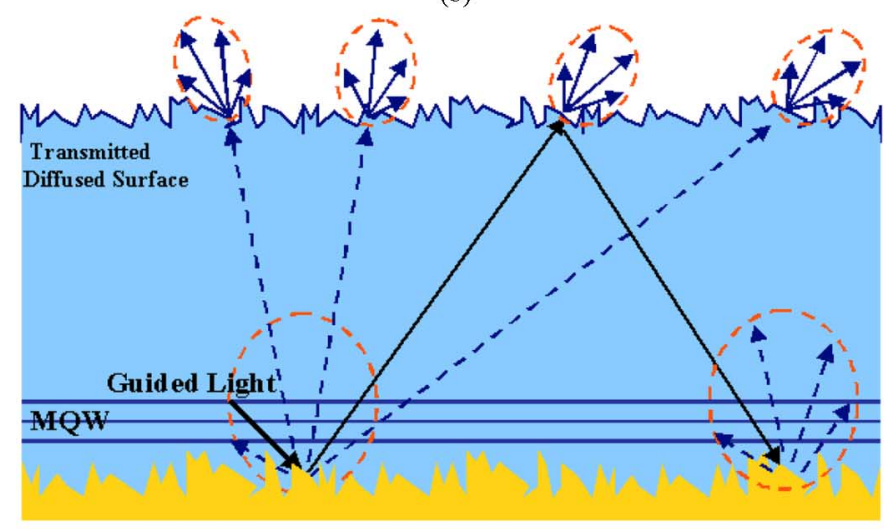

Diffused Reflector

(c)

Fig. 6. Simple optical ray diagrams of (a) conventional LEDs, (b) sample A with top transmitted diffuse surfaces and bottom flat ODRs and (c) sample B with top transmitted diffuse surfaces and bottom diffuse ODRs.

roughened surfaces could easily induce leakage paths. Especially at the procedure of fabricating diffuse ODRs (roughened p-type GaN-ITO-Al), leakage paths may penetrate through $\mathrm{V}$-shape pits to MQWs, degrading LED performances. Therefore, in order to avoid electrical short circuit, specific control of epitaxial condition while growing hexagonal $\mathrm{V}$-shape pits is important. Fig. 7 shows the reverse voltage versus current characteristics of sample A, sample B, and conventional LEDs (each curve represents 10 LED devices average results). It was found that the leakage currents of all LEDs at $-5 \mathrm{~V}$ were in the order of magnitude of $10^{-8} \mathrm{~A}$, indicating the roughening 


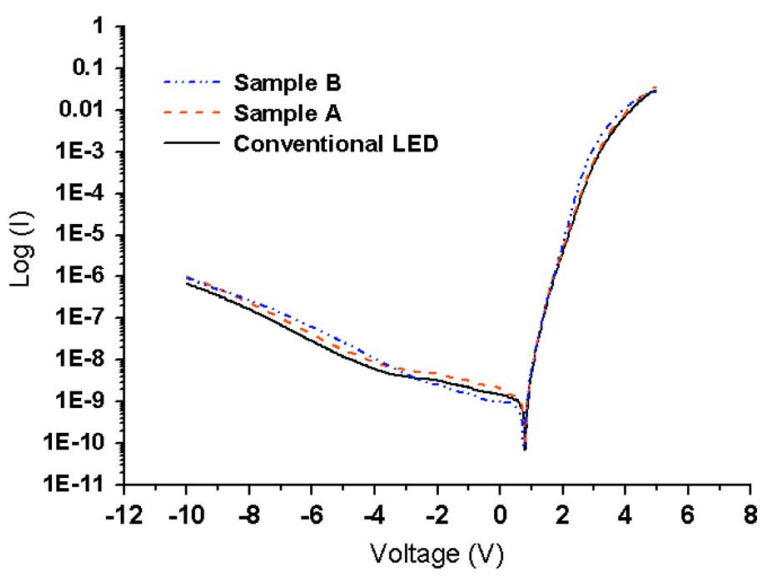

Fig. 7. Leakage current as function of reverse voltage for sample A, sample B, and conventional LEDs.

processes, no matter by epitaxial growth (hexagonal pits) or chemical wet etching (hexagonal cones), did not adversely affect the LED performance.

\section{CONCLUSION}

In conclusion, high light-extraction 465-nm GaN-based vertical LEDs were successfully fabricated with double diffuse surfaces scheme. One surface was a diffusive ODR consisted of an epitaxially roughened p-type GaN layer, an ITO layer, and an Al layer, and another was a wet-chemical etched $N$-face $\mathrm{n}$-GaN transmitted diffuse surface. The external quantum efficiency was calculated to be about $40 \%$. The high performance LED was achieved mainly due to the strong guided-light scattering efficiency while employing double diffuse surfaces. The forward voltages was $3.2 \mathrm{~V}$ at $20 \mathrm{~mA}$ and the leakage currents at $-5 \mathrm{~V}$ was in the order of magnitude of $10^{-8} \mathrm{~A}$, indicating the roughening processes, did not adversely affect the LED performance.

\section{ACKNOWLEDGMENT}

The authors would like to thank Prof. K. M. Lau of Hong Kong University of Science and Technology and Prof. Pilkuhn of National Chiao Tung University for useful discussion.

\section{REFERENCES}

[1] E. Fred Schubert, Light-Emitting Diodes. Cambridge, U.K.: Cambridge Univ. Press.

[2] A. Zukauskas, M. S. Shur, and R. Gaska, Introduction to Solid-State Light. New York: Wiley.

[3] M. Koike, N. Shibata, H. Kato, and Y. Takahashi, "Development of high efficiency GaN-based multiquantum-well light-emitting diodes and their applications," IEEE J. Sel. Topics Quantum Electron., vol. 8, no. 2, pp. 271-277, Mar./Apr. 2002.

[4] M. R. Krames, M. Ochiai-Holcomb, G. E. Höfler, C. Carter-Coman, E. I. Chen, I.-H. Tan, P. Grillot, N. F. Gardner, H. F. Gardner, H. C. Chui, J.-W. Huang, S. A. Stockman, F. A. Kish, and M. G. Craford, "High-power truncated-inverted-pyramid $\left(\mathrm{Al}_{x} \mathrm{Ga}_{1-x}\right)_{0.5} \mathrm{In}_{0.5} \mathrm{P} / \mathrm{GaP}$ light-emitting diodes exhibiting $>50 \%$ external quantum efficiency," Appl. Phys. Lett., vol. 75, pp. 2365-2367, 1999.

[5] Y. J. Lee, H. C. Tseng, H. C. Kuo, S. C. Wang, C. W. Chang, T. C. Hsu, Y. L. Yang, M. H. Hsieh, M. J. Jou, and B. J. Lee, "Improvement in Light-Output Efficiency of AlGaInP LEDs Fabricated on Stripe Patterned Epitaxy," IEEE Photon. Technol. Lett., vol. 17, no. 12, pp. 2532-2534, Dec. 2005.
[6] Y. J. Lee, J. M. Hwang, T. C. Hsu, M. H. Hsieh, M. J. Jou, B. J. Lee, T. C. Lu, H. C. Kuo, and S. C. Wang, "Enhancing output power of GaN-based LEDs grown on chemical wet etching patterned sapphire substrate," IEEE Photon. Technol. Lett., vol. 18, no. 10, pp. 1152-1154, Oct. 2006.

[7] T. Fujii, Y. Gao, R. Sharma, E. L. Hu, S. P. DenBaars, and S. Nakamura, "Increase in the extraction efficiency of GaN-based light-emitting diodes via surface roughening," Appl. Phys. Lett., vol. 84, pp. 855-855, 2004.

[8] T. Gessmann, H. Luo, J.-Q. Xi, K. P. Streubel, and E. F. Schubert, "Light-emitting diodes with integrated omnidirectionally reflective contacts," Proc. SPIE, vol. 5366, pp. 53-61, 2004.

[9] J. K. Kim, H. Luo, Y. Xi, J. M. Shah, T. Gessmann, and E. F. Schubert, "Light extraction in GaInN light-emitting diodes using diffuse omnidirectional reflectors," J. Electrochem. Soc., vol. 153, pp. G105-G107, 2006.

[10] D. I. Florescu, S. M. Ting, J. C. Ramer, D. S. Lee, V. N. Merai, A. Parkeh, D. Lu, E. A. Armour, and L. Chernyak, "Investigation of V-defects and embedded inclusions in InGaN/GaN multiple quantum wells grown by metalorganic chemical vapor deposition on (0001) sapphire," Appl. Phys. Lett., vol. 83, pp. 33-35, 2003.

[11] S. M. Ting, J. C. Ramer, D. I. Florescu, V. N. Merai, B. E. Albert, A. Parkeh, D. S. Lee, D. Lu, D. V. Christini, L. Liu, and E. A. Armour, "Morphological evolution of InGaN/GaN quantum-well heterostructures grown by metalorganic chemical vapor deposition," J. Appl. Phys., vol. 94, pp. 1461-1467, 2003.

[12] Y. K. Su, S. J. Chang, S. C. Wei, S. M. Chen, and W. L. Li, "ESD Engineering of Nitride-Based LEDs," IEEE Trans. Device Mater. Reliab., vol. 5, no. 2, pp. 277-281, Jun. 2005.

[13] S. J. Chang, L. W. Wu, Y. K. Su, Y. P. Hsu, W. C. Lai, J. M. Tsai, J. K. Sheu, and C. T. Lee, "Nitride-based LEDs with $800^{\circ} \mathrm{C}$ grown p-AlInGaN-GaN double-cap layers," IEEE Photon. Technol. Lett., vol. 6, no. 6, pp. 1447-1449, Jun. 2004.

[14] J. T. Chu, T. C. Lu, H. H. Yao, C. C. Kao, W. D. Liang, J. Y. Tsai, H. C. Kuo, and S. C. Wang, "Laser lift-off fabrication of blue-violet GaNbased vertical cavity surface emitting lasers optically pumped at room temperature," Jpn. J. Appl. Phys., vol. V45, no. 4A, pp. 2556-2560, 2006.

[15] Th. Gessmann, Y.-L. Li, E. F. Schubert, J. W. Graff, and J. K. Sheu, "GaInN light-emitting diodes with omni directional reflectors," Proc. SPIE, vol. 4996, pp. 139-144, 2003.

[16] M. G. Craford, "LEDs for solid state lighting and other emerging applications: Status, trends, and challenges," Proc. SPIE, vol. 5941, pp. 594101-1-594101-10, 2005.

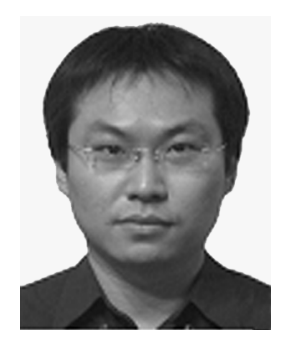

Ya-Ju Lee received the B.S. degree in physics from National Central University, Taiwan, R.O.C., in 2000, and the M.S. degree from the Institute of ElectroOptical Engineering, National Chiao Tung University, Taiwan, R.O.C., in 2002. He is currently working toward the Ph.D. degree in the Department of Photonics and Institute of Electro-Optical Engineering, National Chiao Tung University, Taiwan, R.O.C.

$\mathrm{He}$ is also a Research Engineer in the R\&D Department, Epistar Corporation, Hsinchu, Taiwan, R.O.C.

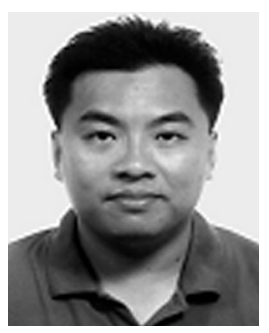

Hao-Chung Kuo (M'98-SM'06) received the B.S. degree in physics from National Taiwan University, Taiwan, R.O.C., the M.S. degree in electrical and computer engineering from Rutgers University, New Brunswick, NJ, in 1995, and the Ph.D. degree from Electrical and Computer Engineering Department, University of Illinois at Urbana Champaign, in 1999.

$\mathrm{He}$ has an extensive professional career both in research and industrial research institutions that includes: Research Consultant in Lucent Technologies, Bell Laboratories (1993-1995); a Senior Research Engineer at Filtronic Solid State (1999-2000); and a Member of Technical Staff in Fiber-Optics Division at Agilent Technologies (2000-2001) and LuxNet Corporation (2001-2002). Since October 2002, he has been with the National Chiao Tung University as a Faculty Member of the Institute of Electro-Optical Engineering. His current research interests include semiconductor lasers, 
VCSELs, blue and UV LED lasers, quantum-confined optoelectronic structures, optoelectronic materials, and high-speed semiconductor devices. He has authored and coauthored 80 internal journal papers and 4 granted patents.

Dr. Kuo is member of SPIE and MRS.

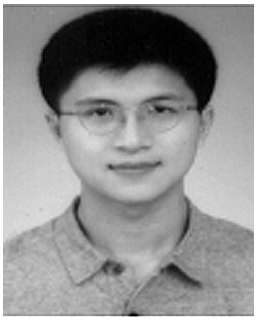

Tien-Chang Lu received the B.S. degree in electrical engineering from National Taiwan University, Taiwan, R.O.C., in 1995, the M.S. degree in electrical engineering from the University of Southern Los Angeles, in 1998, and the Ph.D. degree in electrical engineering and computer science from National Chiao Tung University, Taiwan, R.O.C., in 2004.

$\mathrm{He}$ joined the National Chiao Tung University as a faculty member of Department of Photonics in August 2005.

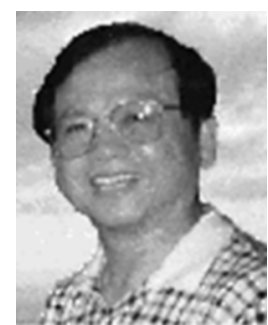

Shing-Chung Wang (M'79-SM'03) received the B.S. degree from National Taiwan University, Taiwan, R.O.C., the M.S. degree from National Tohoku University, Sendai, Japan, and the Ph.D. degree from Stanford University, Stanford, CA, in 1971, all in electrical engineering.

$\mathrm{He}$ has an extensive professional career both in academic and industrial research institutions that includes: a faculty member at National Chiao Tung University (1965-1967); a Research Associate at Stanford University (1971-1974); a Senior Research Scientist at Xerox Corporation (1974-1985); and a Consulting Scientist at Lockheed-Martin Palo Alto Research Laboratories (1985-1995). In 1995, he rejoined National Chiao Tung University, Taiwan, R.O.C., as a faculty member of Institute of Electro-Optical Engineering. His current research interests include semiconductor lasers, VCSELs, blue and UV lasers, quantum-confined optoelectronic structures, optoelectronic materials, diode-pumped lasers, and semiconductor laser applications.

Prof. Wang is a Fellow of the Optical Society of America and a recipient of Outstanding Scholar Award from the Foundation for the Advancement of Outstanding Scholarship. 